OPEN ACCESS

Edited by:

Kwangcheol Casey Jeong,

University of Florida, United States

Reviewed by:

Young Min Kwon,

University of Arkansas, United States

Robert J. Moore,

RMIT University, Australia

${ }^{*}$ Correspondence:

Baosheng Liu

liubaosh@163.com

Specialty section:

This article was submitted to

Systems Microbiology,

a section of the journal

Frontiers in Microbiology

Received: 30 December 2018

Accepted: 28 May 2019

Published: 18 June 2019

Citation:

Zhu N, Wang J, Yu L, Zhang Q, Chen K and Liu B (2019) Modulation of Growth Performance and Intestinal Microbiota in Chickens Fed Plant

Extracts or Virginiamycin.

Front. Microbiol. 10:1333.

doi: 10.3389/fmicb.2019.01333

\section{Modulation of Growth Performance and Intestinal Microbiota in Chickens Fed Plant Extracts or Virginiamycin}

\author{
Nianhua Zhu' ${ }^{1}$, Jun Wang ${ }^{1}$, Longfei Yu' ${ }^{1}$, Qiman Zhang ${ }^{2}$, Kai Chen ${ }^{1}$ and Baosheng Liu${ }^{1 *}$ \\ ${ }^{1}$ College of Animal Science and Technology, Jiangxi Agricultural University, Nanchang, China, ${ }^{2}$ Guangdong Ruisheng \\ Technology Co., Ltd., Guangzhou, China
}

In this study, the effects of plant extracts (PEs) and virginiamycin (VIRG) on broiler growth performance, as well as on host intestinal microbiota composition and function were investigated. A total of 288 one-day-old male Cobb broiler chickens were randomly divided into four treatment groups (with six replicates per group). The duodenal, ileal, and cecal content of six broilers per treatment group after 14 and 28 days of treatment were sampled. This material was used for high-throughput Illumina sequencing of the V3-V4 region of the 16S rRNA gene. The results showed that chickens fed $400 \mathrm{mg} / \mathrm{kg}$ plant extracts (HPE group) had significantly higher average body weights at day 28 as compared to the control group (CT; $P<0.05$ ), and lower feed-to-meat ratios over days $15-42(P<0.01)$. Within the HPE group at day 14 , the relative abundances of two bacterial phyla and 10 bacterial genera increased significantly in the ileal microbiota, and the relative abundance of three bacterial phyla and four bacterial genera decreased. The relative abundance of the genus Lactobacillus in the cecal microbiota decreased from $21.48 \%$ (CT group) to $8.41 \%$ (fed $200 \mathrm{mg} / \mathrm{kg}$ PEs; LPE group), $4.2 \%$ (HPE group), and $6.58 \%$ (fed $30 \mathrm{mg} / \mathrm{kg}$ virginiamycin; VIRG group) after 28 days. In contrast, Faecalibacterium and unclassified Rikenellaceae increased in abundance in the HPE group (from 18 to $28.46 \%$ and from 10.83 to $27.63 \%$, respectively), while Bacteroides (36.7\%) and Lachnospiraceae increased in abundance in the VIRG group. PICRUSt function analysis showed that the ileal microbiota of the PE treatment groups were more enriched in genes related to the meolism of cofactors and vitamins. In addition, the cecal microbiotas of the LPE and HPE groups were enriched in genes predicted to encode enzymes within 15 and 20 pathways, respectively. These pathways included protein digestion and absorption, amino acid metabolism, lipid biosynthesis, lipopolysaccharide biosynthesis, the citrate cycle (TCA cycle), and lipoic acid metabolism. Similarly, the VIRG group was enriched in 55 metabolic pathways (17 in the duodenum, 18 in the ileum, and 20 in the cecum) on day $28(P<0.05)$. Thus, the results indicated that the observed increase in broiler growth performance after PE or VIRG supplementation might be attributed to an improvement in intestinal microbial composition and metabolic function.

\footnotetext{
Keywords: plant extracts, virginiamycin, intestinal microbiota, growth performance, broilers
} 


\section{INTRODUCTION}

Subtherapeutic levels of antibiotics have been widely used in the swine and poultry industries to improve weight gain and feed conversion efficiency (Cromwell, 2002; Dibner and Richards, 2005). However, there has been increasing concern about the health and environmental safety risks of this practice, particularly due to the persistence of antibiotic residues and the transmission of antibiotic resistance. For these reasons, the use of antibiotics as feed additives has been restricted or even banned in many countries (Diarra and Malouin, 2014). Recent studies have therefore attempted to develop alternative approaches with which to maintain production performance and reduce mortality in the poultry industry (Salaheen et al., 2017). Among the available alternatives, plant extracts (PEs) or phytogenic additives have been identified as appropriate candidates due to their safety and effective antibacterial behavior (Diaz Carrasco et al., 2016; Ran et al., 2016).

Plant extracts, are essential oils that are extracted from plant materials (e.g., flowers, leaves, roots, and fruits) via solvent extraction or steam distillation. PEs are primarily composed of terpenes, terpene derivatives, and mixtures of various other compounds (Baser and Demirci, 2007; Brenes and Roura, 2010; Li et al., 2018). Due to the complexity of the PE compounds, the extracts have diverse biological effects (Diaz Carrasco et al., 2016). For example, some PE treatments have been shown to increase body weight gain and decrease the feed-to-gain ratio of broilers (Jamroz and Kamel, 2002; Ciftici et al., 2005; Jang et al., 2007; Windisch et al., 2008; Zhang et al., 2009; Khattak et al., 2014; Zeng et al., 2015). Although the specific mechanisms by which PEs improve animal growth performance have yet to be elucidated, it is clear that some PEs reduce pathogenic stress in the gut, increase digestive enzyme secretions, improve nutrient absorption, act as antioxidants, and support host immunity; these properties help to explain beneficial effects of PEs observed in the livestock industries (Windisch et al., 2008; Awaad et al., 2014; Wlodarska et al., 2015; Zeng et al., 2015). The well-known antibacterial properties of PEs have been widely tested against a wide range of pathogenic bacteria using cultures, denaturing gradient gel electrophoresis (DGGE), or PCR (Brenes and Roura, 2010; Yin et al., 2017). Recently, Li et al. (2018) used highthroughput $16 \mathrm{~S}$ rRNA sequencing methods to investigate the effects of dietary supplementation with carvacrol and thymol on the microbial composition and metabolites in colons of weaned piglets. However, studies of the effects of PEs on the growth performance and the correlation between it and the change of intestinal microbiotas in chickens are limited.

Although the underlying mechanisms by which antibiotics enhance animal performance remain unclear, the effects of antibiotics on the host gut microbiota have been established (Pourabedin et al., 2015). Importantly, it was shown that antibiotics improved animal growth by regulating the intestinal microbiota (Diarra and Malouin, 2014). Therefore, in the present study, it was hypothesized that PEs and/or VIRG would improve broiler growth performance by modulating the composition and function of the host intestinal microbiota. This study aimed to investigate the effects of PEs and VIRG on broiler growth performance and intestinal microbiota, using high-throughput Illumina sequencing of the $16 \mathrm{~S}$ rRNA gene.

\section{MATERIALS AND METHODS}

\section{PE Chemical Composition}

A commercial PE product was used, which contained $20 \%$ carvacrol and $25 \%$ thymol as active components, 35\% silicon dioxide as a caking inhibitor, and $12 \%$ glycerides as stabilizing agents (Guangdong Ruisheng Technology Co., Ltd., Guangdong, China).

\section{Experimental Animals, Diets, and Treatments}

This study was approved by the Animal Care and Use Committee of China Agricultural University, permit number SYXK20171208. A total of 288 one-day-old male Cobb 500 broiler chickens were randomly divided into four groups, with six replicates per group ( $n=12$ chickens per replicate). Each group was fed one of the following four diets: CT, the basal diet, without any added growth promoter; VIRG, the basal diet supplemented with virginiamycin (30 mg/kg); LPE, the basal diet supplemented with $200 \mathrm{mg} / \mathrm{kg}$ PEs; or HPE, the basal diet supplemented with $400 \mathrm{mg} / \mathrm{kg}$ PEs. The components and nutritional composition of the basal diets are given in

TABLE 1 | Dietary compositions and nutrient levels of broilers (as-fed basis).

\begin{tabular}{lccc}
\hline Ingredient (\%) & $\begin{array}{c}\text { Starter } \\
\text { (1-14 days) }\end{array}$ & $\begin{array}{c}\text { Grower } \\
\text { (15-28 days) }\end{array}$ & $\begin{array}{c}\text { Finisher } \\
\text { (29-42 days) }\end{array}$ \\
\hline Corn & 55.00 & 57.65 & 59.03 \\
Soybean meal & 38.00 & 34.87 & 33.80 \\
Soybean oil & 3.00 & 3.50 & 4.00 \\
Dicalcium phosphate & 1.85 & 1.60 & 1.70 \\
Limestone & 1.17 & 1.20 & 1.30 \\
Salt & 0.35 & 0.35 & 0.35 \\
Vitamin premix & 0.03 & 0.03 & 0.03 \\
Choline chloride (50\%) & 0.10 & 0.07 & 0.07 \\
Mineral premix ${ }^{2}$ & 0.20 & 0.20 & 0.18 \\
DL-Met & 0.25 & 0.16 & 0.09 \\
HCl-Lys & 0.05 & 0.04 & 0.03 \\
Calculated nutrition levels, \% & & & 0.95 \\
ME, MJ/Kg & 12.16 & 12.78 & 12.82 \\
CP & 20.74 & 19.60 & 18.00 \\
Met + Cys & 0.92 & 1.05 & 0.64 \\
Lys & 1.1 & 0.95 & 0.9 \\
Ca & 1.08 & 0.95 & 0.9 \\
P & 0.71 & 0.64 & \\
\hline & & & \\
\hline
\end{tabular}

${ }^{1}$ Vitamin premix containing the following per kilogram of diet: vitamin A, 10,000 IU; vitamin $D_{3}$ (cholecalciferol), 3,500 IU; vitamin E (DL- $\alpha$-tocopheryl acetate), $60 \mathrm{mg}$; vitamin $\mathrm{K}$ (menadione), $3 \mathrm{mg}$; thiamine, $3 \mathrm{mg}$; riboflavin, $6 \mathrm{mg}$; pyridoxine, $5 \mathrm{mg}$; vitamin $B_{12}$ (cyanocobalamin), $0.01 \mathrm{mg}$; niacin, $45 \mathrm{mg}$; pantothenic acid (D-calcium pantothenate), $11 \mathrm{mg}$; folic acid, $1 \mathrm{mg}$; biotin, $0.15 \mathrm{mg}$; choline chloride, $500 \mathrm{mg}$; ethoxyquin (antioxidant), $150 \mathrm{mg} .{ }^{2}$ Mineral premix containing the following per kilogram of diet: Fe, 60 mg; Mn, 100 mg; Zn, 60 mg; Cu, 10 mg; I, 1 mg; Co, $0.2 \mathrm{mg}$; Se, $0.15 \mathrm{mg}$. 
Table 1. The basal diets met the nutrient requirements of the National Research Council [NRC] (1994).

\section{Chicken Management and Growth Performance}

Chickens were reared in battery pens $(1.0 \times 0.8 \times 0.4 \mathrm{~m})$ with plastic wire floors and two water nipples per pen. Chickens had access to food and water ad libitum. The room temperature was maintained at $32^{\circ} \mathrm{C}$ for the first week, reduced evenly over the next week to $24^{\circ} \mathrm{C}$, and maintained at $24^{\circ} \mathrm{C}$ from day 14 until the end of the experiment. The daily photoperiod was $20 \mathrm{~h}$ light: $4 \mathrm{~h}$ dark, and chickens were conventionally vaccinated against the Newcastle disease virus on day 7 . Feed intake and weight gain for each replicate were measured on days 14, 28, and 42 . The feed conversion ratio (FCR) was calculated for days 0-14, 15-28, and 29-42.

\section{Sample Collection}

On days 14 and 28, six chickens per treatment group (one bird per replicate) were randomly selected, and each selected bird was intravenously injected with sodium pentobarbital (30 mg/kg body weight). Chickens were killed using jugular exsanguinations, and $2 \mathrm{~g}$ of digesta from the duodenum, ileum, and cecum of each bird was taken. The digesta samples were mixed, collected in sterilized tubes, immediately frozen in liquid nitrogen, and stored at $-80^{\circ} \mathrm{C}$.

\section{DNA Extraction}

Total bacterial genomic DNA was extracted from each digesta sample using a FastDNA SPIN extraction kit (MP Biomedicals, Santa Ana, CA, United States), following the manufacturer's instructions. DNA quantity and quality were determined using a NanoDrop ND-1000 spectrophotometer (Thermo Fisher Scientific, Waltham, MA, United States) and agarose gel electrophoresis respectively. DNA samples were stored at $-20^{\circ} \mathrm{C}$.

\section{S rDNA Amplicon Pyrosequencing}

The V3-V4 region of the bacterial 16S rRNA genes was PCR amplified using the universal primers 338F $\left(5^{\prime}\right.$-ACTCCTAC GGGAGGCAGCA-3') and 806R (5'-GGACTACHVGGGTWT CTAAT-3'), following She et al. (2018). Briefly, sample-specific 7-bp barcodes were incorporated into the primers for multiplex sequencing. Each PCR was performed in a $25-\mu 1$ volume containing $2 \mu \mathrm{L}$ DNA template, forward and reverse primers, dNTPs, DNA Polymerase, reaction buffer, and $\mathrm{ddH}_{2} \mathrm{O}$. The cycling conditions were an initial denaturation at $98^{\circ} \mathrm{C}$ for $2 \mathrm{~min}$; 25 cycles of $15 \mathrm{~s}$ at $98^{\circ} \mathrm{C}, 30 \mathrm{~s}$ at $55^{\circ} \mathrm{C}$, and $30 \mathrm{~s}$ at $72^{\circ} \mathrm{C}$; and a final extension of $5 \mathrm{~min}$ at $72^{\circ} \mathrm{C}$. PCR amplicons were purified with Agencourt AMPure Beads (Beckman Coulter, Indianapolis, IN, United States), and quantified using the PicoGreen dsDNA Assay Kit (Invitrogen, Carlsbad, CA, United States). Purified amplicons were pooled in equal amounts, and then paired-end sequenced on an Illlumina MiSeq platform using the MiSeq Reagent Kit v3 at Shanghai Personal Biotechnology Co., Ltd. (Shanghai, China).

\section{Sequence Processing and Analysis}

The sequencing data were processed using the Quantitative Insights Into Microbial Ecology (QIIME, v1.8.0) pipeline, following Caporaso et al. (2010). Briefly, raw sequencing reads with complete barcode matches were assigned to the appropriate sample and identified as valid, Sequences $<150$ bp long, with average Phred scores $<20$, containing ambiguous bases, or with mononucleotide repeats longer than 8 bp were considered lowquality and excluded from further analysis (Gill et al., 2006; Chen and Jiang, 2014). Paired-end reads were assembled using FLASH (Magoč and Salzberg, 2011). After chimera detection with QIIME, UCLUST (Edgar, 2010) was used to group the remaining high-quality sequences into operational taxonomic units (OTUs) based on a minimum sequence identity of $97 \%$. A representative sequence was identified for each OTU using default parameters. The OTUs were taxonomically classified using BLAST; each representative sequence was searched the against the Greengenes Database (DeSantis et al., 2006) and the best hit was selected (Altschul et al., 1997). An OTU table was used to record the abundance and taxonomic affiliation of each OTU in each sample. OTUs representing less than $0.001 \%$ of all of the sequences across all of the samples were ignored. To maintain a constant sequencing depth across all of the samples, an averaged, rounded, rarefied OTU table was generated by re-sampling an average of 100 OTU subsets at a minimum sequencing depth of $90 \%$. This averaged OTU table was used for all of the subsequent analyses.

\section{Bioinformatics Analysis}

Bioinformatics analyses of the sequence data were primarily performed in QIIME and R. v3.2.0. OTU-level alpha diversity indices, such as the Chao1 richness estimator, Shannon diversity index, Simpson index, and Abundance-based Coverage Estimator (ACE), were calculated based on the OTU table in QIIME, and OTU-level rank abundance curves were generated to compare the evenness and richness of OTUs among samples. The numbers of OTUs unique and shared among samples or groups, irrespective of abundance, were visualized using the VennDiagram R package (Zaura et al., 2009). The relative abundances of the recovered phyla, classes, orders, families, genera and species were statistically compared among samples and groups using Metastats (White et al., 2009). Linear discriminant analysis effect size (LEfSe) analysis was performed with default parameters in order to detect differentially abundant taxa across groups (Segata et al., 2011). Microbial functions were predicted using Phylogenetic Investigation of Communities by Reconstruction of Unobserved States (PICRUSt), based on highquality sequences (Langille et al., 2013). OTUs were normalized by copy number, and metagenomic prediction was performed based on Kyoto Encyclopedia of Genes and Genomes (KEGG) (Kanehisa et al., 2012).

\section{Statistical Analysis}

Data are shown as mean and SEM. Differences in average body weight, average daily gain (ADG), FCR, alpha diversity indices, and the relative abundance of bacterial taxa among treatment 
groups were analyzed using one-way ANOVAs, followed by Duncan multiple comparison tests, in SPSS v18.0 (Chicago, IL, United States). The relative abundances of the 10 most abundant phyla, the relative abundances of genera and species, and bacterial functional pathways were analyzed using $\mathrm{LEfSe}^{1}$ (Segata et al., 2011). The alpha value of the Kruskal-Wallis test among classes was set to 0.5 , and the $\log 10$ linear discriminant analysis (LDA) score was limited to 2.0 .

\section{Data Access}

All raw sequences were deposited in the NCBI Sequence Read Archive (accession number SRP188289).

\section{RESULTS}

\section{Growth Performance}

PE or VIRG dietary supplementation affected broiler growth performance (Table 2). On day 28, chicken body weight in the HPE group was higher (1264.3 g) than that in the CT group $(1076.1 \mathrm{~g})(P<0.05)$. On $\mathrm{d} 42$, the final body weight of broilers treated with HPE (2536.57 g) and VIRG (2520.77 g) was increased 4.66 and $4.03 \%$ respectively as compared to that of the CT group $(2423.0 \mathrm{~g})$. However, the difference was insignificant $(P=0.20)$. Treatment with PEs or VIRG significantly decreased the feedto-gain ratio over days $15-28$ and $29-42(P<0.01)$. However, there were no significant differences in body weight and the feed-to-gain ratio over days $1-14(P>0.05)$.

${ }^{1}$ http://huttenhower.sph.harvard.edu/galaxy

TABLE 2 | Effects of plant extracts and virginiamycin on broiler growth performance ${ }^{1}$.

\begin{tabular}{lcccccc}
\hline & CT & VIRG & LPE & HPE & SEM & P-value \\
\hline Average body weight & & & & & & \\
at 14 days (g/bird) & 306.26 & 298.22 & 308.18 & 312.60 & 4.0 & 0.68 \\
at 28 days (g/bird) & $1076.1^{\mathrm{b}}$ & $1120^{\mathrm{b}}$ & $1172.7^{\mathrm{ab}}$ & $1264.3^{\mathrm{a}}$ & 22.0 & 0.01 \\
at 42 days (g/bird) & 2423 & 2520.7 & 2504.3 & 2536.6 & 20.2 & 0.20 \\
Average daily gain (g/d) & & & & & & \\
Days 1-14 & 19 & 18 & 19 & 19 & 0.288 & 0.51 \\
Days 15-28 & $55^{\mathrm{c}}$ & $58^{\mathrm{c}}$ & $60^{\mathrm{ab}}$ & $68^{\mathrm{a}}$ & 1.636 & 0.03 \\
Days 29-42 & $96^{\mathrm{ab}}$ & $100^{\mathrm{a}}$ & $97^{\mathrm{ab}}$ & $91^{\mathrm{b}}$ & 1.495 & 0.19 \\
Average feed intake (g/d) & & & & & & \\
Days 1-14 & 27 & 24 & 27 & 30 & 0.014 & 0.72 \\
Days 15-28 & $92^{\mathrm{a}}$ & $84^{\mathrm{b}}$ & $86^{\mathrm{b}}$ & $91^{\mathrm{c}}$ & 0.031 & 0.02 \\
Days 29-42 & $156^{\mathrm{a}}$ & $134^{\mathrm{b}}$ & $141^{\mathrm{b}}$ & $150^{\mathrm{a}}$ & 0.044 & 0.04 \\
Feed to gain ratio & & & & & & \\
Days 1-14 & 1.46 & 1.38 & 1.30 & 1.36 & 0.060 & 0.68 \\
Days 15-28 & $1.74^{\mathrm{a}}$ & $1.44^{\mathrm{c}}$ & $1.56^{\mathrm{bc}}$ & $1.60^{\mathrm{b}}$ & 0.050 & 0.001 \\
Days 29-42 & $1.86^{\mathrm{a}}$ & $1.65^{\mathrm{b}}$ & $1.64^{\mathrm{b}}$ & $1.64^{\mathrm{b}}$ & 0.081 & 0.001 \\
\hline
\end{tabular}

${ }^{1}$ Each value represents the mean of six replicates. CT, basal diet (control); VIRG, basal diet supplemented with $30 \mathrm{mg} / \mathrm{kg}$ virginiamycin; LPE, basal diet supplemented with $200 \mathrm{mg} / \mathrm{kg}$ plant extracts; HPE, basal diet supplemented with $400 \mathrm{mg} / \mathrm{kg}$ plant extracts. ${ }^{a, b}$ Different letters in the same row indicate significant differences between the respective means $(P<0.05)$

\section{Diversity and Structure of Intestinal Microbiota}

Raw reads were denoised and then cleaned to remove chimeras and low-quality sequences. A total of 5,254,194 quality-controlled reads were obtained, an average of 36,487 sequences per sample. An average of 1080 OTUs per sample (based on $97 \%$ sequence similarity) was identified, with an average sequence length of 494.5 bp. The bacterial OTU numbers, microbial richness, and diversity are presented in Table 3. In the HPE group, the Shannon and Simpson diversity indices in the ileum were significantly lower than those of the CT group on day 14 only $(P<0.05$; Table 3). However, in the VIRG group, the Shannon and Simpson diversity indices in the duodenum were significantly greater than those of the CT group on days 14 and $28(P<0.05$; Table 3). No significant differences in richness and diversity were observed in the ceca among all of the treatments groups on days 14 and 28 $(P>0.05)$. Principal coordinate analysis indicated that microbes from the ceca of the HPE and CT groups formed distinct clusters, while microbes from both the ileum and ceca of the VIRG and CT groups formed distinct clusters (Figure 1). No differences in duodenal microbial communities among treatment groups were identified on days 14 and 28 (Figures 1A,B).

\section{Relative Abundance of Bacterial Taxa in the Intestinal Microbiota}

Firmicutes was the dominant bacterial phylum in duodenum and ileum (75-95\% of all OTUs; Figure 2A). In the cecum, the microbiota included 55-70\% Firmicutes, 25-30\% Bacteroidetes, and $3 \%$ other phyla. Dietary treatments affected relative bacterial abundance at the phylum level (Figure 2A). Compared with the CT group, Firmicutes was more abundant in the intestinal microbiota of the HPE group on day 14 . On day 28, Bacteroidetes was more abundant in the cecal microbiota of the HPE group as compared to the CT group, and Actinobacteria was less abundant. However, Firmicutes was less abundant in the intestinal microbiota of the VIRG treatment group as compared to the control group (except in the duodenum on day 14). In addition, VIRG treatment increased the relative proportion of Proteobacteria in the ileum and Bacteroidetes in the cecum.

Lactobacillus was the dominant genus in the duodenal and ileal microbial communities (Figure 2B). On day 14, the duodenal microbiotas of the broilers were 50-70\% Lactobacillus and $20-27 \%$ Pseudomonas. On day 28 , almost $90 \%$ of all bacteria in the duodenum and ileum samples were Lactobacillus. The cecal microbiota included 10-20\% Lactobacillus and 10-20\% Faecalibacterium. The SPSS analysis of all bacterial genera with relative abundances $>0.1 \%$ is shown in Table 4 . The results indicated that 28 days of dietary supplementation with PEs significantly decreased the relative abundance of Lactobacillus (from $21.48 \%$ in the CT group to 8.41 and $4.2 \%$ in the LPE and HPE groups, respectively; $P<0.05$ ), and significantly increased the relative abundances of Faecalibacterium (from 18-20\% in the CT group to $28-31 \%$ in the HPE group; $P<0.01$ ) and Rikenellaceae (from $10.83 \%$ in the CT group to $25-27 \%$ in the LPE and HPE groups; $P<0.01)$ in the cecal microbiota. Dietary supplementation with VIRG for 14 and 28 days also significantly 
TABLE 3 | Effects of plant extracts and virginiamycin on the diversity of the intestinal microbiota ${ }^{1}$.

\begin{tabular}{|c|c|c|c|c|c|c|c|c|}
\hline & & & CT & VIRG & LPE & HPE & SEM & $P$-value \\
\hline \multirow{8}{*}{ Day 14} & & Chao1 & 1121 & 1017 & 918 & 968.4 & 29.22 & 0.07 \\
\hline & & Simpson & $0.87^{\mathrm{ab}}$ & $0.96^{a}$ & $0.86 a^{b}$ & $0.79^{b}$ & 0.02 & 0.03 \\
\hline & & Shannon & $6.13^{b}$ & $7.10^{a}$ & $5.81^{b}$ & $5.38^{b}$ & 0.20 & 0.01 \\
\hline & & ACE & 1399 & 1286 & 1236.65 & 1093 & 43.83 & 0.09 \\
\hline & & Simpson & $0.93^{a}$ & $0.93^{a}$ & $0.90^{\mathrm{a}}$ & $0.82^{b}$ & 0.02 & 0.02 \\
\hline & & Shannon & $6.99^{a}$ & $7.06^{a}$ & $6.49^{\mathrm{ab}}$ & $5.61^{b}$ & 0.19 & 0.01 \\
\hline & Cecum & OTU & 1366 & 1101 & 1278 & 1397 & 56.67 & 0.26 \\
\hline & & Chao1 & 1516 & 1185 & 1455 & 1574 & 66.77 & 0.17 \\
\hline \multirow{10}{*}{ Day 28} & & Chao1 & 745.9 & 951 & 760 & 967 & 58.18 & 0.39 \\
\hline & & ACE & 809.5 & 1004 & 818 & 1044 & 62.68 & 0.44 \\
\hline & & Simpson & $0.47^{\mathrm{b}}$ & $0.82^{\mathrm{a}}$ & $0.57^{\mathrm{b}}$ & $0.65^{\mathrm{ab}}$ & 0.05 & 0.03 \\
\hline & & Shannon & $2.99^{b}$ & $5.84^{a}$ & $3.64^{b}$ & $4.41^{\mathrm{ab}}$ & 0.38 & 0.04 \\
\hline & Ileum & OTU & 945.2 & 736 & 1062 & 901 & 43.81 & 0.06 \\
\hline & & Chao1 & 1066 & 819 & 1175 & 1020 & 48.02 & 0.05 \\
\hline & & $\mathrm{ACE}$ & $1144^{a}$ & $858^{b}$ & $1240^{a}$ & $1094^{\mathrm{ab}}$ & 51.36 & 0.04 \\
\hline & & Simpson & 0.76 & 0.86 & 0.83 & 0.69 & 0.03 & 0.20 \\
\hline & & Shannon & 5.25 & 5.81 & 6.04 & 4.68 & 0.27 & 0.31 \\
\hline & Cecum & OTU & 1256 & 1215 & 1070 & 1147 & 38.96 & 0.37 \\
\hline
\end{tabular}

${ }^{1}$ Each value represents the mean of six replicates. CT, basal diet (control); VIRG, basal diet supplemented with $30 \mathrm{mg} / \mathrm{kg}$ virginiamycin; LPE, basal diet supplemented with $200 \mathrm{mg} / \mathrm{kg}$ plant extracts; HPE, basal diet supplemented with $400 \mathrm{mg} / \mathrm{kg}$ plant extracts. ${ }^{a, b}$ Different letters in the same row indicate significant differences between the respective means $(P<0.05)$.

decreased the relative abundance of Lactobacillus in the broiler ileum and cecum samples as compared to the CT group, and increased the relative abundance of Bacteroides in the cecum samples $(P<0.01)$. Supplementation with either VIRG or PEs also increased the relative abundance of Lachnospiraceae in the cecum of broilers on day 14 .

The LEfSe results suggested that supplementation with PEs modified the microbial composition of ileum on day 14 and that of cecum on day 28 (Figure 3; LDA > 2; $P<0.05)$. On day 14 , the relative abundances of three phyla (Firmicutes, Bacteroidetes and Thermi) and four genera (e.g., Candidatus Arthromitus and unclassified Burkholderiales) increased in the ileal microbiotas of the HPE group as compared to the CT group, and the relative abundances of two phyla (Proteobacteria and Tenericutes) and 10 genera (e.g., Ruminococcus, unclassified RF39, Faecalibacterium, and Oscillospira) decreased. On day 28, the relative abundances of two phyla (Bacteroidetes and Cyanobacteria) and three genera (Alistipes, unclassified Rikenellaceae, unclassified
Mogibacteriaceae, and Anaeroplasma) increased in the cecal microbiota of the HPE group as compared to the CT group, and the relative abundances of one phylum (Actinobacteria) and two genera (Lactobacillus and unclassified Coriobacteriaceae) decreased (Figures 3A,B). In addition, the relative abundances of Bacteroidetes and several genera of unclassified Rikenellaceae increased in the cecal microbiotas of the LPE group on day 28 as compared to the CT group (Figure 3D). On day 14, the relative abundances of the genera Ruminococcus, Lachnospiraceae, and Oscillospira were higher in the cecal microbiota of the VIRG group as compared to the CT group (Figure 3E). On day 28, the relative abundances of one phylum (Firmicutes) and four genera (Lactobacillus, unclassified RF39, unclassified Rikenellaceae, and unclassified Erysipelotrichaceae) were lower in the duodenal and cecal microbiotas of the VIRG group as compared to the CT group, and the relative abundances of one phylum (Bacteroidetes) and four genera (Bacteroides, Enterobacteriaceae, Ruminococcus, and Lachnospiraceae) were higher in the 

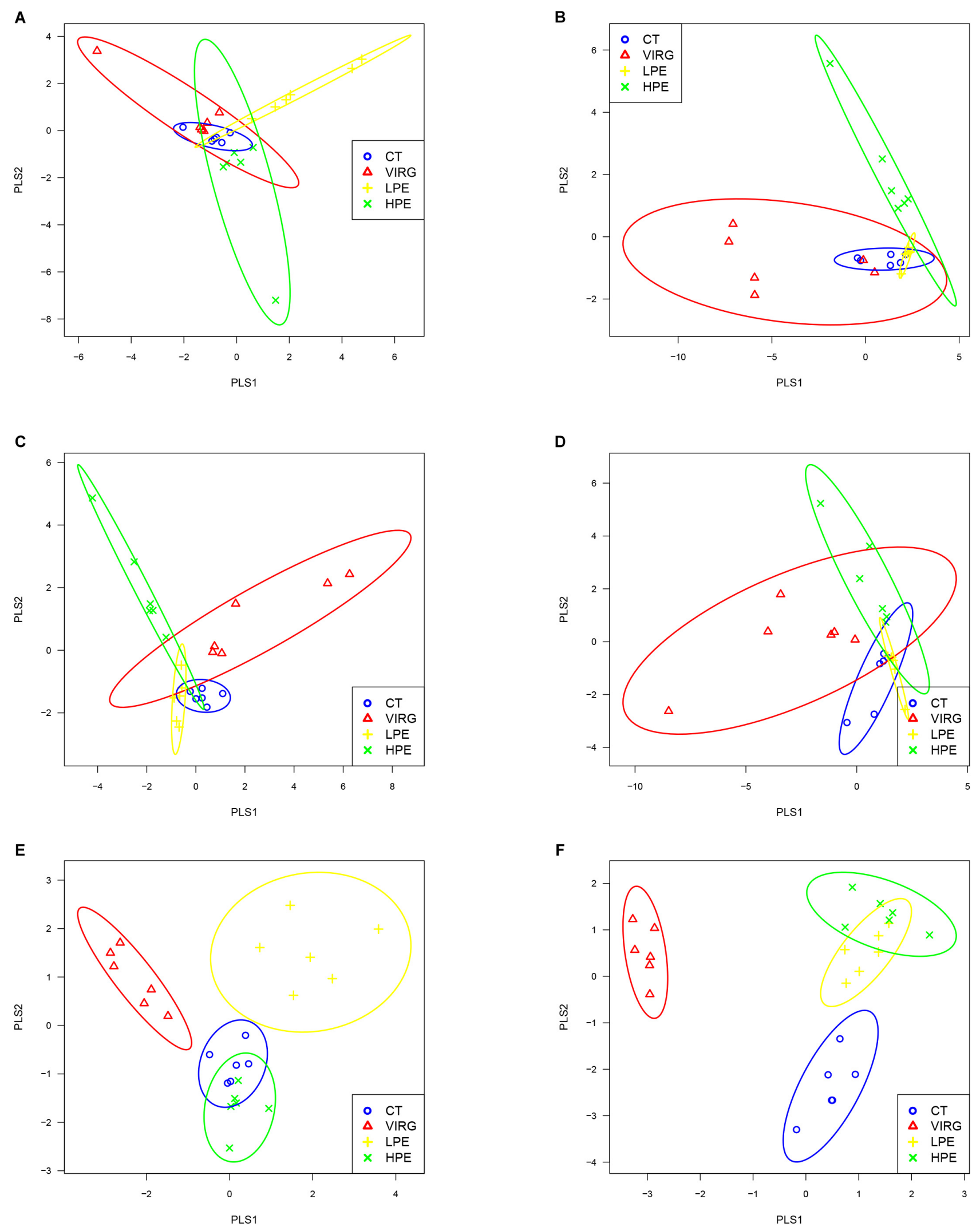

FIGURE 1 | Continued 
FIGURE 1 | Principal coordinate analysis (PLS-DA), based on the weighted unifrac distances of $16 S$ rRNA bacterial sequences from the intestines of broilers. (A,B) PLS-DA of duodenal bacteria at (A) day 14 or (B) day 28. (C,D) PLS-DA of ileal bacteria at (C) day 14 or (D) day 28. (E,F) PLS-DA of cecal bacteria at (E) at day 14 or (F) day 28. CT, basal diet (control); VIRG, basal diet supplemented with $30 \mathrm{mg} / \mathrm{kg}$ virginiamycin; LPE, basal diet supplemented with $200 \mathrm{mg} / \mathrm{kg}$ plant extracts; HPE, basal diet supplemented with $400 \mathrm{mg} / \mathrm{kg}$ plant extracts.

A

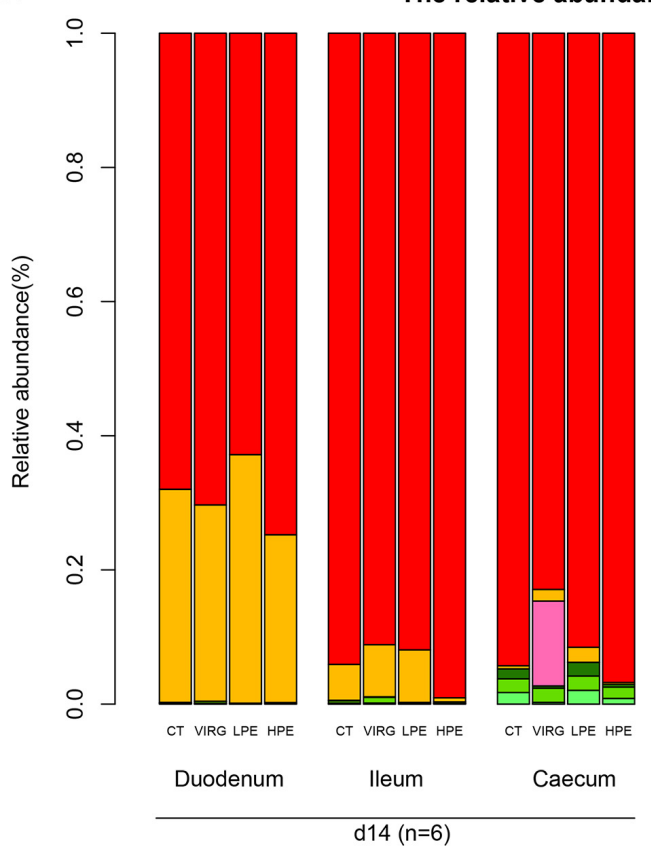

The relative abundance of bacteria at phylum level

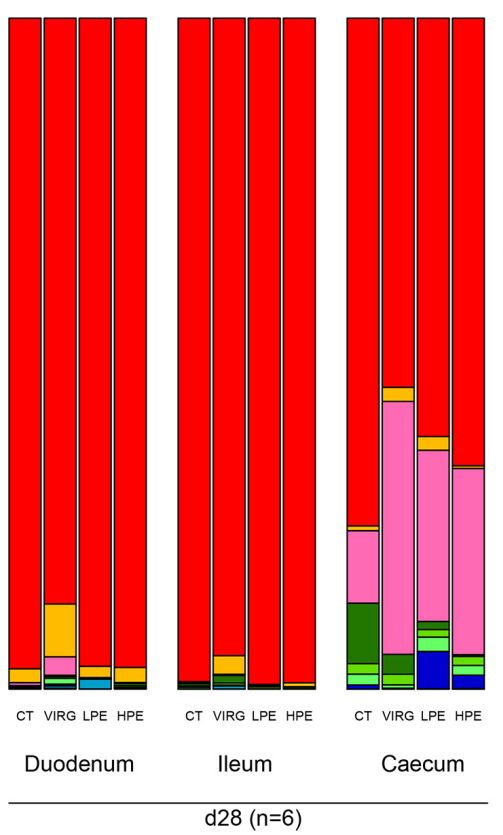

Firmicutes

$\square$ Proteobacteria

$\square$ Bacteroidetes

$\square$ Actinobacteria

$\square$ Other

$\square$ Tenericutes

- Verrucomicrobia

$\square$ Cyanobacteria

$\square$ [Thermi]
B

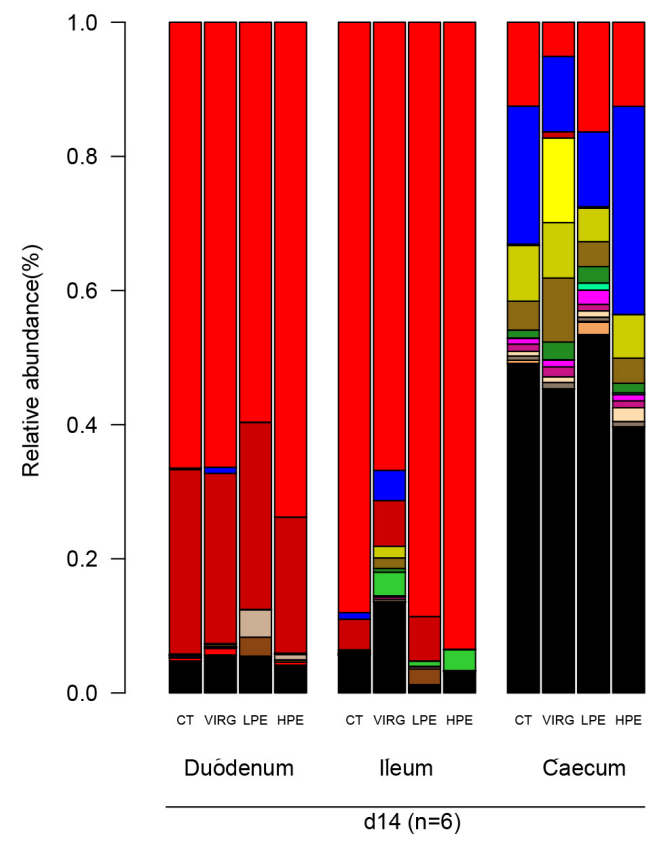

The relative abundance of bacteria at genus level

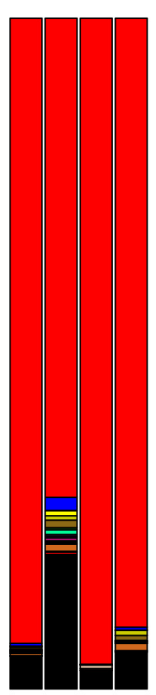

CT VIRG LPE HPE

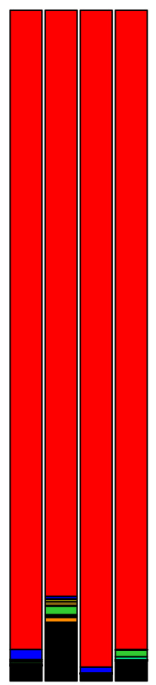

CT VIRG LPE HPE

Ileum

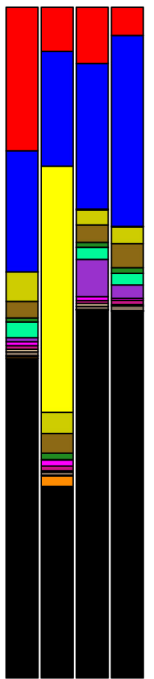

CT VIRG LPE HPE

Caecum $\square$ Lactobacillus

- Faecalibacterium

- Pseudomonas

$\square$ Bacteroides

$\square$ [Ruminococcus]

$\square$ Oscillospira

$\square$ Ruminococcus

$\square$ Candidatus Arthromitus

$\square$ Streptococcus

$\square$ Akkermansia

$\square$ Blautia

$\square$ Dorea

$\square$ Coprobacillus

$\square$ Acinetobacter

$\square$ Coprococcus

$\square$ Clostridium

$\square$ Cupriavidus

$\square$ Butyricicoccus

$\square$ Bifidobacterium

$\square$ Anoxybacillus

- Other

FIGURE 2 | Effects of plant extracts and virginiamycin on duodenal, ileal, and cecal microbiota composition at phylum and genus level. Relative abundances of bacterial phyla (A) and genera (B) in four treatment groups. CT, basal diet (control); VIRG, basal diet supplemented with $30 \mathrm{mg} / \mathrm{kg}$ virginiamycin; LPE, basal diet supplemented with $200 \mathrm{mg} / \mathrm{kg}$ plant extracts; HPE, basal diet supplemented with $400 \mathrm{mg} / \mathrm{kg}$ plant extracts. Intestinal chyme was collected from six birds per group for this analysis $(n=6)$. 
TABLE 4 | Effects of plant extracts and virginiamycin on the relative abundance of bacterial genera (\%) $)^{1}$.

\begin{tabular}{|c|c|c|c|c|c|c|c|}
\hline \multirow[t]{2}{*}{ Genus } & \multirow[t]{2}{*}{ Treatment } & \multicolumn{3}{|c|}{ Day 14} & \multicolumn{3}{|c|}{ Day 28} \\
\hline & & Duodenum & lleum & Cecum & Duodenum & Ileum & Cecum \\
\hline \multirow{3}{*}{ Lactobacillus } & VIRG & 66.35 & 66.55 & 5.13 & 71.21 & 87.3 & 6.58 \\
\hline & LPE & 59.63 & 88.6 & 16.45 & 96.33 & 97.9 & 8.41 \\
\hline & HPE & 73.8 & 93.5 & 12.61 & 90.73 & 95.3 & 4.2 \\
\hline \multirow[t]{5}{*}{ Faecalibacterium } & CT & 0.21 & 0.983 & 20.53 & 0.4 & 1.51 & 18 \\
\hline & VIRG & 0.9 & 4.63 & 11.25 & 1.98 & 0.4 & 17.13 \\
\hline & LPE & 0.033 & $N D^{2}$ & 11.25 & 0.16 & 0.86 & 21.71 \\
\hline & HPE & 0.033 & ND & 31 & 0.41 & 0.06 & 28.46 \\
\hline & $P$-value & 0.461 & 0.457 & 0.117 & 0.057 & 0.607 & 0.413 \\
\hline \multirow[t]{2}{*}{ Unclassified Ruminococcaceae } & СТ & 0.3 & 2.98 & 24.21 & 1 & 0.43 & 10.38 \\
\hline & $P$-value & 0.514 & 0.229 & 0.648 & 0.258 & 0.455 & 0.98 \\
\hline \multirow[t]{5}{*}{ Pseudomonas } & CT & 27.51 & 4.58 & 0.183 & $\mathrm{ND}$ & ND & ND \\
\hline & VIRG & 25.36 & 6.8 & 0.933 & ND & 0.022 & ND \\
\hline & LPE & 27.91 & ND & 0.2 & ND & ND & ND \\
\hline & HPE & 20.26 & 0.067 & 0 & ND & ND & ND \\
\hline & $P$-value & 0.635 & 0.104 & 0.473 & & 0.013 & \\
\hline \multirow[t]{5}{*}{ Unclassified Rikenellaceae } & СТ & 0.016 & ND & ND & ND & ND & 10.83 \\
\hline & VIRG & ND & ND & ND & ND & ND & 0.88 \\
\hline & LPE & 0.016 & ND & ND & ND & ND & 25.43 \\
\hline & HPE & ND & ND & ND & ND & ND & 27.63 \\
\hline & $P$-value & 0.582 & & & & & 0.0001 \\
\hline \multirow{3}{*}{ Unclassified Lachnospiraceae } & LPE & ND & ND & 5.51 & 0.016 & ND & 3.2 \\
\hline & HPE & ND & ND & 6.06 & 0.76 & ND & 2.5 \\
\hline & $P$-value & 0.396 & 0.212 & 0.045 & & & 0.027 \\
\hline
\end{tabular}

${ }^{1}$ Each value represents the mean of six replicates. CT, basal diet (control); VIRG, basal diet supplemented with $30 \mathrm{mg} / \mathrm{kg}$ virginiamycin; LPE, basal diet supplemented with $200 \mathrm{mg} / \mathrm{kg}$ plant extracts; HPE, basal diet supplemented with $400 \mathrm{mg} / \mathrm{kg}$ plant extracts. ${ }^{2} \mathrm{ND}$ indicates a relative abundance $<0.01 \%$.

cecal microbiotas of the VIRG group as compared to the CT group (Figure 3F).

\section{Changes in Intestinal Microbiota Function}

The predicted functions of the intestinal microbiota were identified using PICRUSt. At KEGG level 2 (Figure 4), several functions of the ileal microbiota were differentially enriched among treatments after 14 days, including cellular processes and signaling, metabolism of cofactors and vitamins, and infectious diseases. Several functions of the cecal microbiota were also differentially enriched on day 14 , including the xenobiotics biodegradation metabolism and enzyme families (Figure 4A). Several functions associated with the digestive system and metabolic disease were differentially enriched in the intestinal microbiota among treatments after 28 days. In the VIRG group at days 14 and 28 , the ileal and cecal microbiotas were enriched in functions associated with the carbohydrate metabolism and the digestive system (Figure 4B).

The LEfSe analysis of KEGG level 3 identified 145 KEGG categories related to metabolic pathways; of these 40 were significantly differentially enriched among treatment groups (LDA $>2 ; P<0.05)$. The heat map of metabolic pathway enrichment across treatment groups showed that the amino 

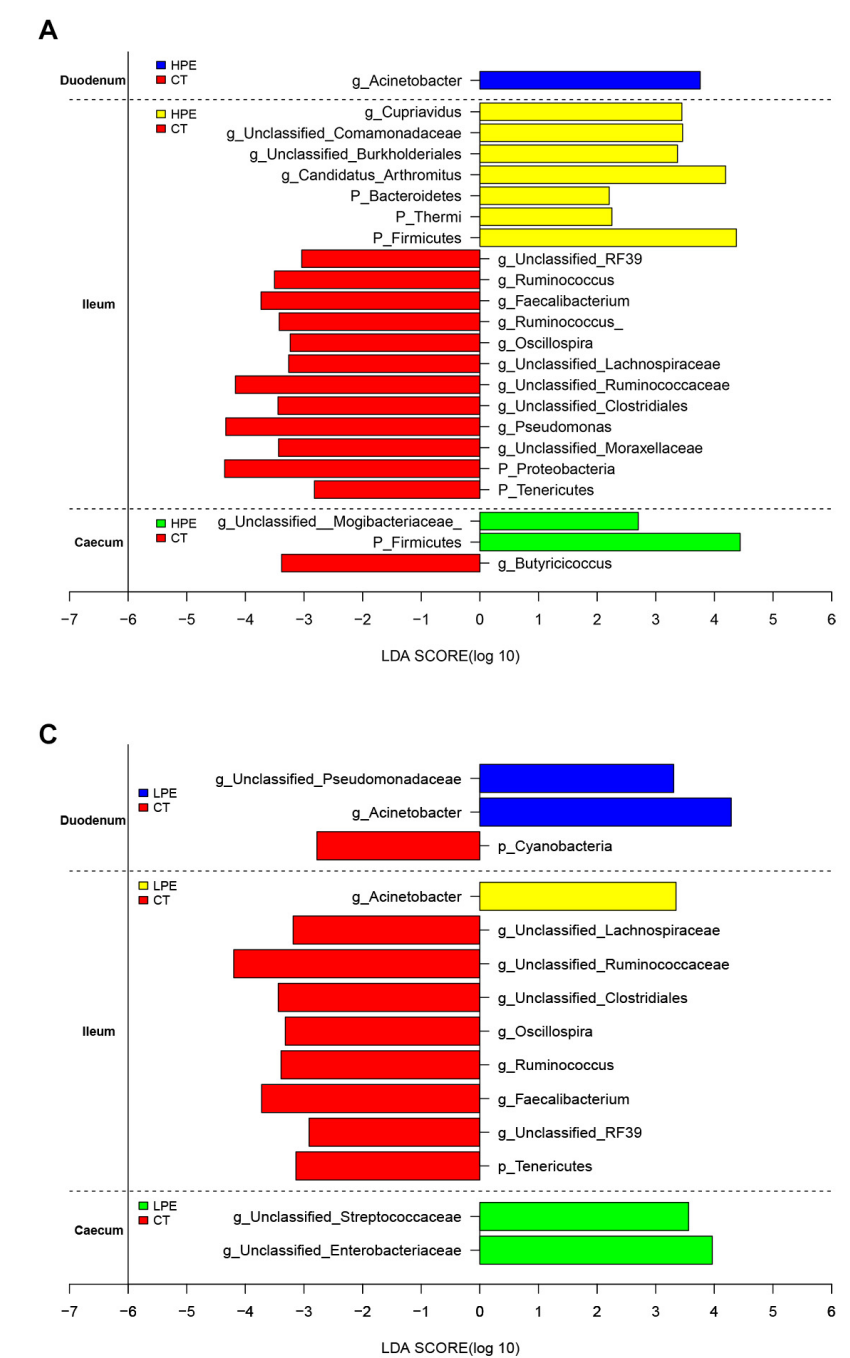

E

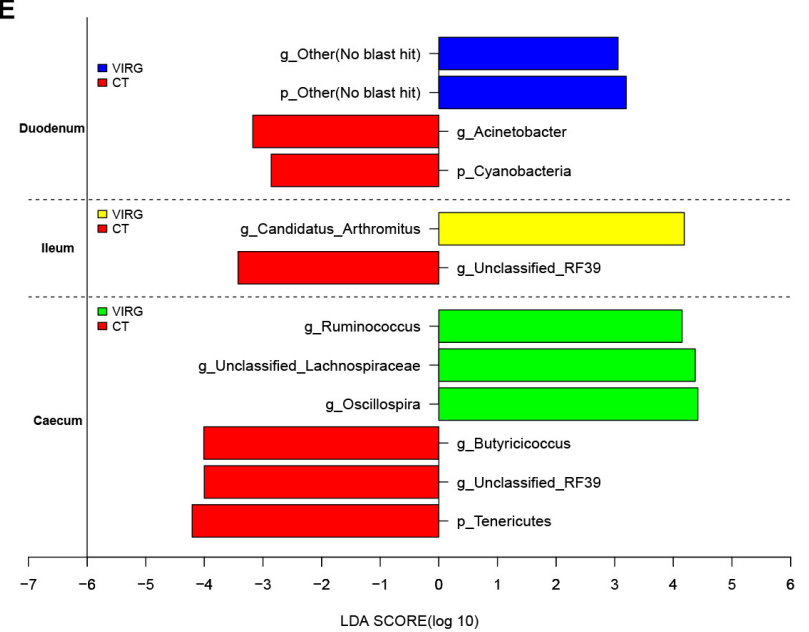

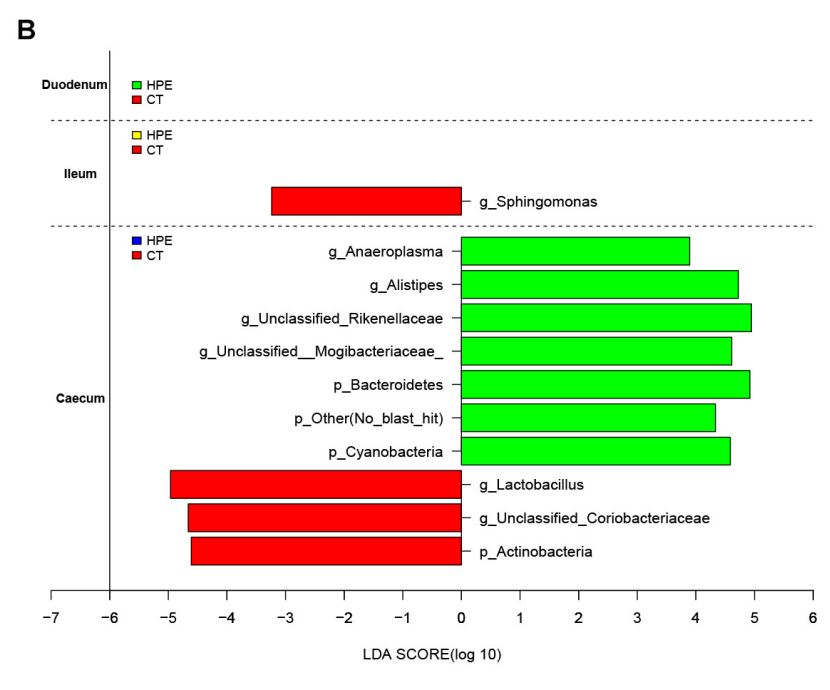

D

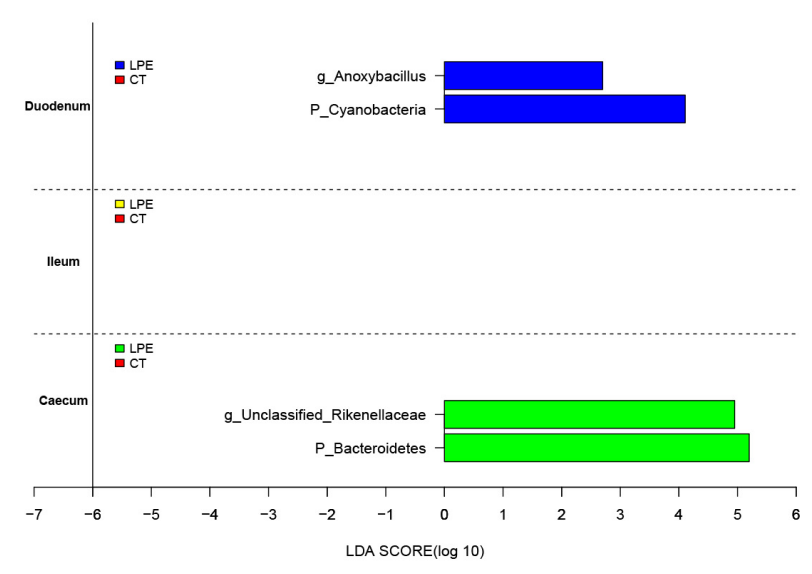

$\mathbf{F}$

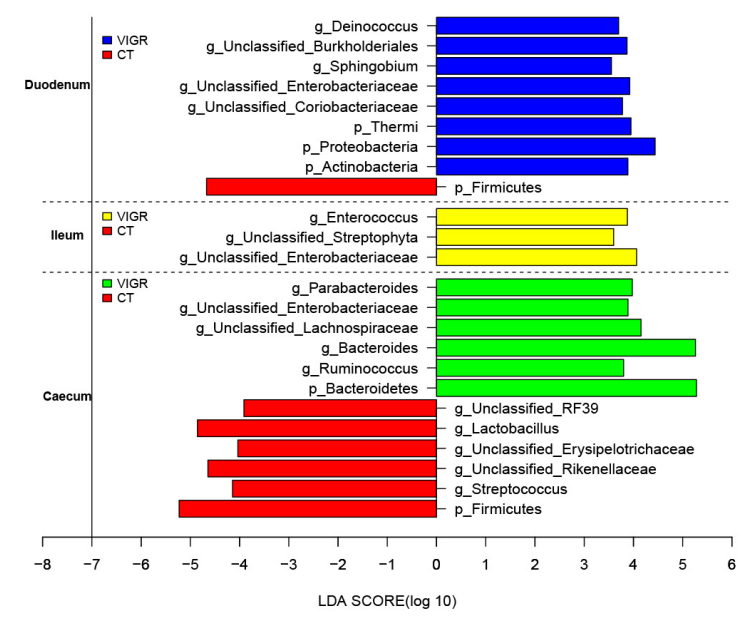

FIGURE 3 | Histograms showing the results of the linear discriminant analysis effect size (LEfSe) measurements, identifying the most differentially abundant phyla and genera between the PENIRG and CT groups. (A,B) Representation of the LEfSe results, showing different phyla and genera in the duodenum, ileum, and cecum samples from the HPE and CT chickens at day 14 and 28. (C,D) Representation of the LEfSe results, showing different phyla and genera in the duodenum, ileum, 
FIGURE 3 | Continued

and cecum samples from the LPE and CT chickens at day 14 and 28. (E,F) Representation of the LEfSe results, showing different phyla and genera in the duodenum, ileum, and cecum samples from the VIRG and CT chickens at day 14 and 28. The CT chickens are indicated with a negative LDA scores (LDA > 2; $P<0.05)$. CT, basal diet (control); VIRG, basal diet supplemented with $30 \mathrm{mg} / \mathrm{kg}$ virginiamycin; LPE, basal diet supplemented with $200 \mathrm{mg} / \mathrm{kg}$ plant extracts; HPE, basal diet supplemented with $400 \mathrm{mg} / \mathrm{kg}$ plant extracts.

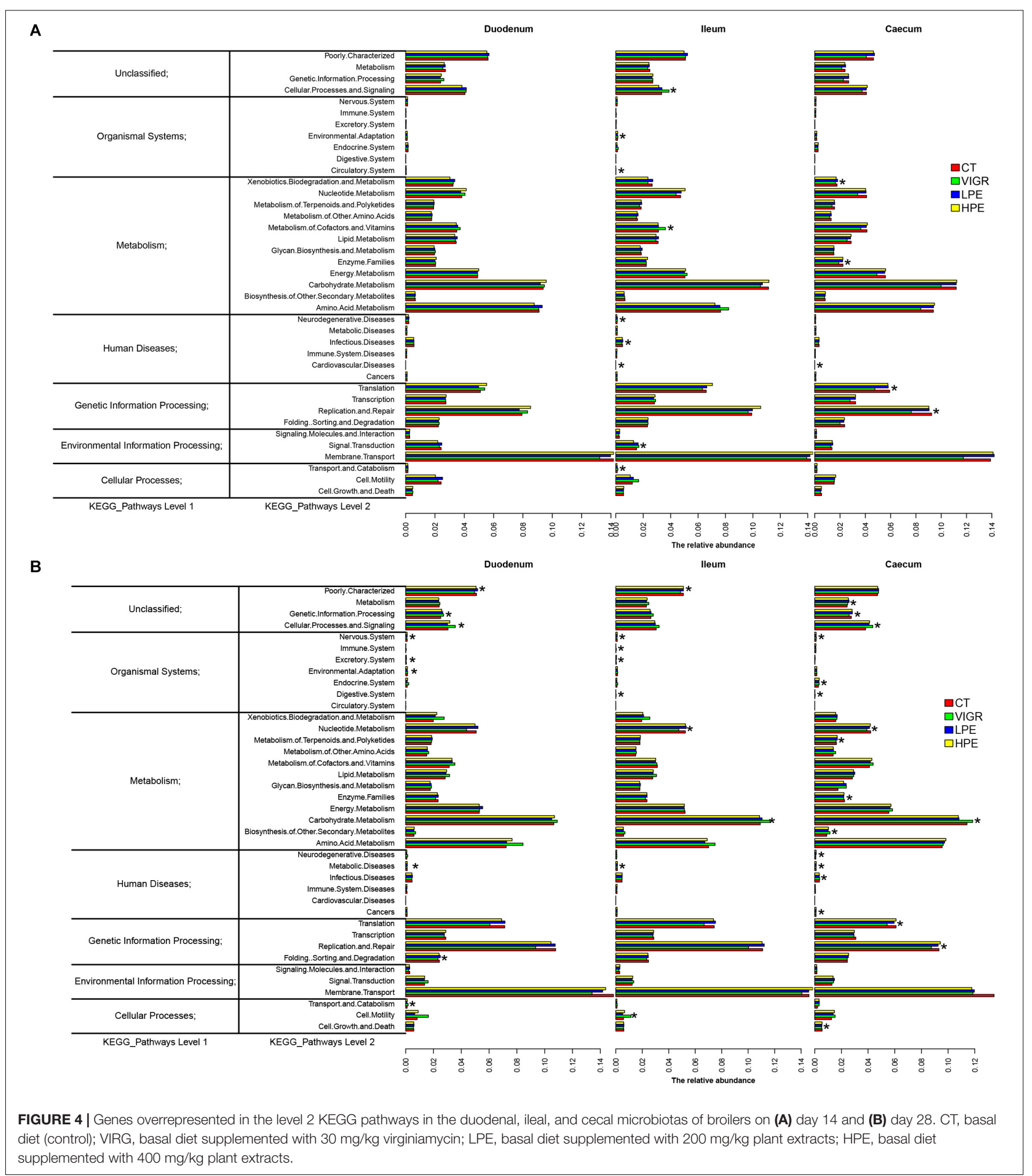




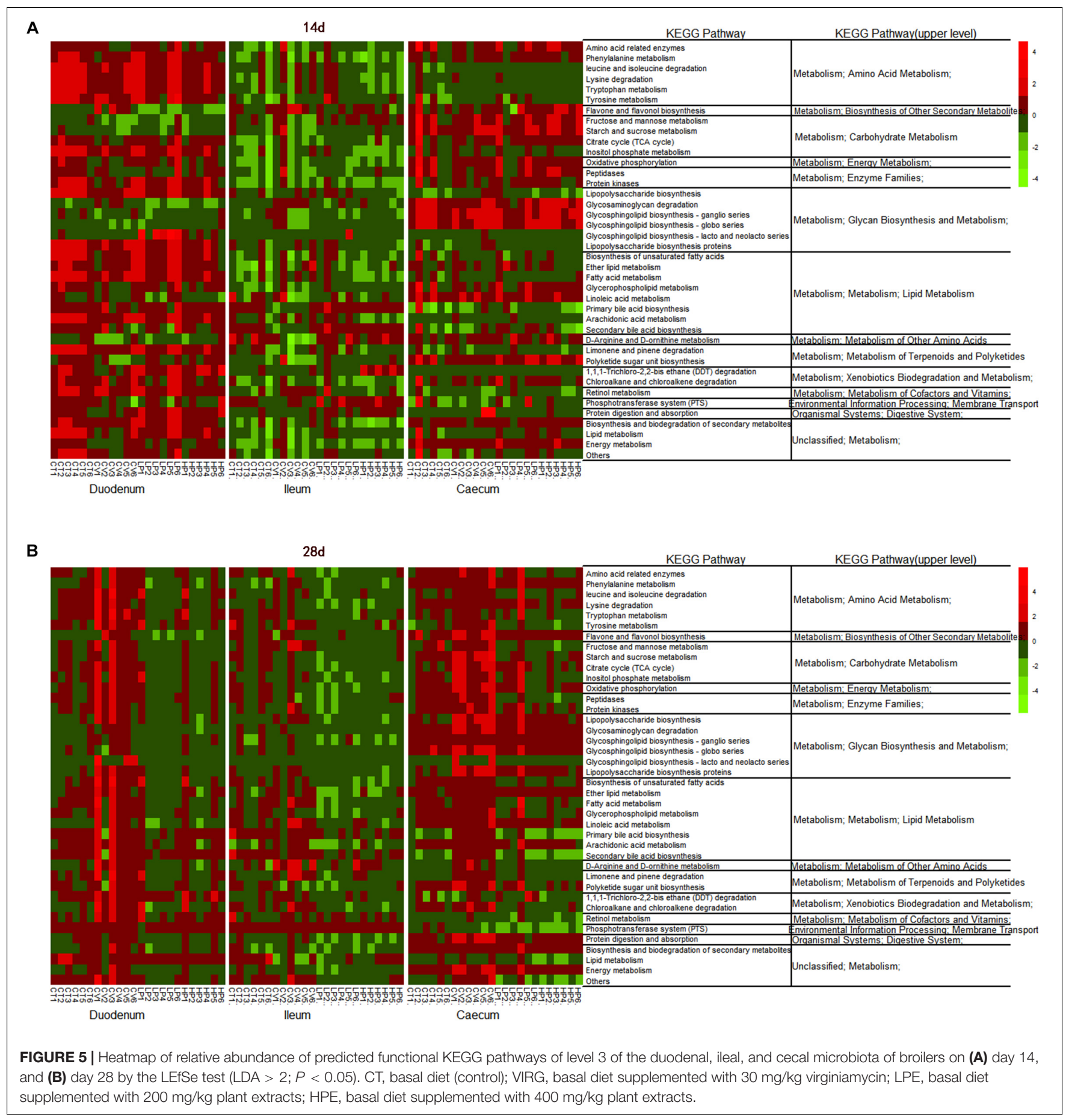

acid and lipid metabolisms in the duodenum samples were significantly affected in the HPE and VIRG groups (Figure 5). In the HPE group at day 14, the cecal microbiota was more enriched in functions involved in amino-acid-related enzymes, the D-Arginine and D-ornithine metabolism and carbohydrate metabolism (i.e., fructose and mannose metabolism, starch and sucrose metabolism, and the citrate cycle), as compared to the CT group (Figure 5A). In the VIRG group at day 28, the duodenal and cecal microbiotas were more enriched in functions involved in the amino acid, lipid, and carbohydrate metabolisms than those of the CT group (Figure 5B).

LEfSe was used to analyze differences in the enrichment of 145 identified level-3 metabolic pathways between the $\mathrm{CT}$ and HPE/VIRG groups (Figure 6). At day 14 in the ileal microbiota of the HPE group, functions associated with the cysteine and methionine metabolism, the citrate cycle (TCA cycle), and the selenocompound metabolism were enriched compared to the CT group (Figure 6A). 

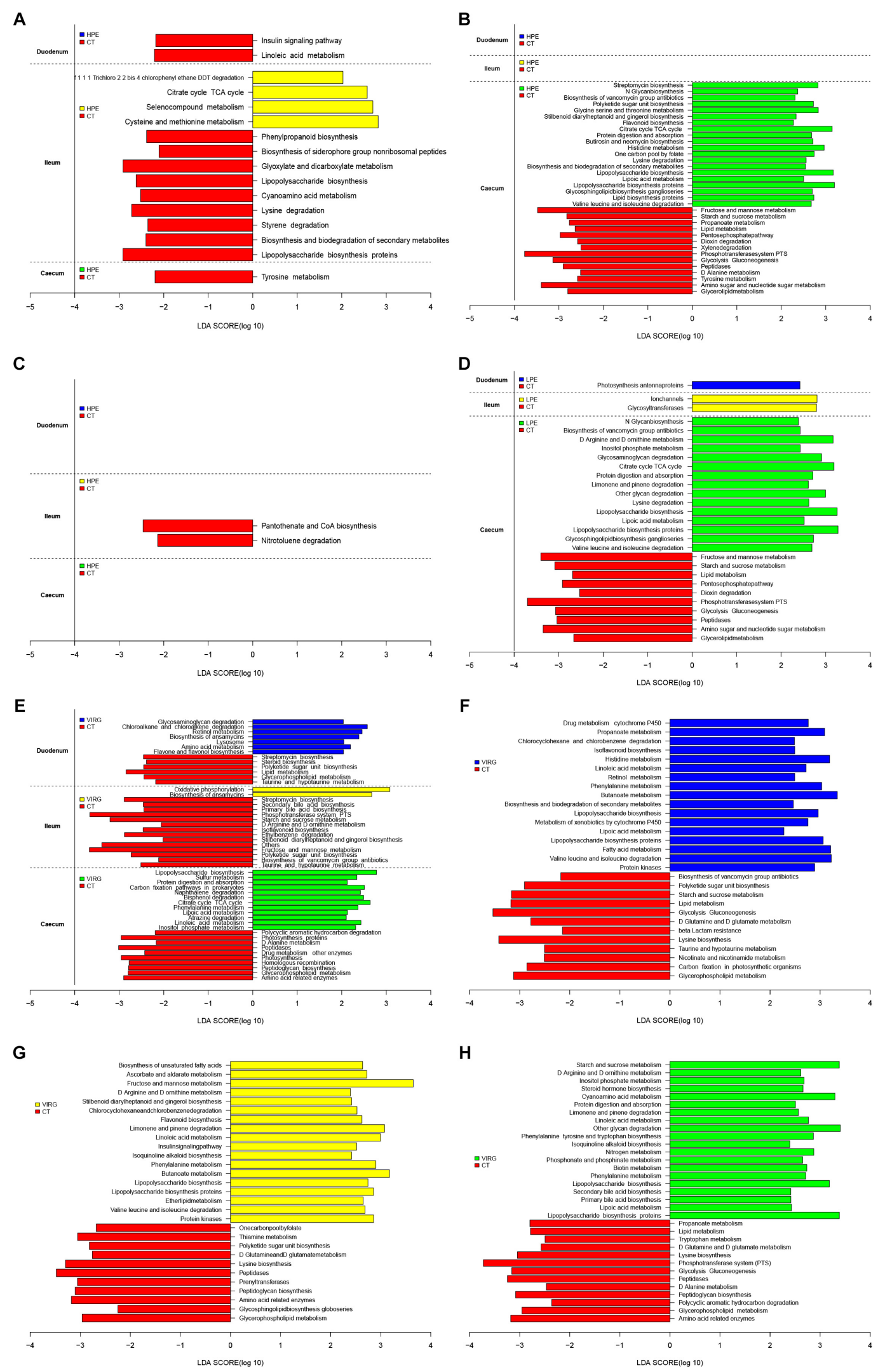

FIGURE 6 | Continued 
FIGURE 6 | PICRUSt analysis of differences in metabolic functions among treatments, based on LEfSe results (LDA > 2; $P<0.05)$. (A,B) Differences in the metabolic functions of the intestinal microbiota between HPE and CT group chickens at (A) day 14 and (B) day 28. (C,D) Differences in the metabolic functions of the intestinal microbiota between LPE and CT group chickens at (C) day 14 and (D) day 28. (E) Differences in the metabolic functions of the intestinal microbiota between VIRG and CT group chickens at day 14. (F-H) Differences in the metabolic functions of the (F) duodenal, (G) ileal, and (H) cecal microbiota between VIRG and CT group chickens at day 28. CT, basal diet (control); VIRG, basal diet supplemented with 30 mg/kg virginiamycin; LPE, basal diet supplemented with $200 \mathrm{mg} / \mathrm{kg}$ plant extracts; HPE, basal diet supplemented with $400 \mathrm{mg} / \mathrm{kg}$ plant extracts.

At day 28, 20 metabolic pathways were more enriched in the HPE cecal microbiota as compared to the CT cecal microbiota (Figure 6B), including protein digestion/absorption, various metabolisms (including amino acid, lipid, carbohydrate, histidine, glycine, serine and threonine, lysine, valine, and lipoic acid), leucine and isoleucine degradation, lipid biosynthesis proteins, and the citrate cycle (TCA cycle). In the LPE group at 28 days, 15 metabolic pathways were enriched in the cecal microbiota (Figures 6C,D). Interestingly, pathways associated with protein digestion and absorption, amino acid metabolism, lipopolysaccharide biosynthesis proteins, the citrate cycle (TCA cycle), and lipoic acid metabolism were upregulated in both the LPE and HPE groups.

In the intestinal microbiota of the VIRG group at day 14, 21 identified metabolic pathways were enriched as compared to the CT group ( 7 in the duodenum, 2 in the ileum, and 12 in the cecum; Figure 6E). In addition, 55 categories (17 in the duodenum, 18 in the ileum, and 20 in the cecum) were significantly enriched in the VIRG group on day 28 (Figure 6F). Thus, dietary supplementation with PEs and VIRG affected important predicted functions of the intestinal microbiota.

\section{DISCUSSION}

The present study showed that treatment with PEs increased the ADGs of broiler chickens during the growth phase, and improved conversion feed rates during all of the phases. Several previous studies have shown that PEs have positive effects on body weight gain and the feed-to-gain ratio in chickens (Jamroz and Kamel, 2002; Ciftici et al., 2005; Jang et al., 2007; Zhang et al., 2009: Khattak et al., 2014). However, some authors have found that PE treatment only improved chicken body weight gain, and did not affect the feed-to-gain ratio (Ocak et al., 2008; Bozkurt et al., 2009). Finally, Jamroz et al. (2005) and Amad et al. (2011) showed that PEs improved the feed conversion rate, but had no effect on body weight or feed intake.

Although it remains unclear precisely how PEs supplementation improves chicken growth, several mechanisms have been proposed, including modifications of intestinal microbial ecology (e.g., reducing pathogenic stress or increasing the abundance of beneficial microorganism in the gut), increasing digestive enzyme secretions, and improving nutrient absorption (Windisch et al., 2008; Awaad et al., 2014; Wlodarska et al., 2015; Zeng et al., 2015; Stevanović et al., 2018). In vitro experiments have shown that PEs have antibacterial, antifungal, and antiviral properties; PEs are more active against gram-positive bacteria than against gram-negative bacteria (Burt, 2004; Windisch et al., 2008; Zeng et al., 2015). Some recent in vivo experiments have suggested that PEs modify the composition of the intestinal microbiota, increasing the relative abundance of Firmicutes in the gut (Salaheen et al., 2017; Li et al., 2018), as well as the abundance of Clostridiales, Ruminococcaceae, and Lachnospiraceae in the PE-treatment group (Diaz Carrasco et al., 2018). Here, the Simpson and Shannon diversity indices in the ileum microbiota decreased in the HPE group. This was consistent with the results of Yin et al. (2017), which showed that supplementation with essential oils decreased the Shannon and Simpson diversity indices. Moreover, HPE treatment increased the relative abundances of Firmicutes in duodenal and ileal microbiotas, and increased the relative abundances of Bacteroidetes and several genera (unclassified Rikenellaceae, Faecalibacterium and Lachnospiraceae) in the cecal microbiota.

Recent studies have identified correlations between intestinal microbial composition and the efficiency of energy extraction in humans and animals (Turnbaugh et al., 2006; Ley et al., 2007). Firmicutes and Bacteroidetes are two of the dominant bacterial phyla in chicken. The ratio of Firmicutes abundance to Bacteroidetes abundance in the gut microbiota has been linked to the efficiency of energy harvesting in humans and various animals (Turnbaugh et al., 2006; Ley et al., 2007; Zhao et al., 2015). Firmicutes species are associated with the decomposition of polysaccharides and the production of butyrate. Bacteroidetes species degrade complex carbohydrates and synthesize propionate via the succinate pathway (Turnbaugh et al., 2006; Louis et al., 2014). Alistipes and unclassified Rikenellaceae fall into Bacteroidales. Alistipes, belong to the main member within family Rikenellaceae, are generally considered beneficial to the host gut (Abe et al., 2012). These bileresistant bacteria have saccharolytic and proteolytic properties, produce acetic acid by producing fibrinolysin, digest gelatin, and ferment carbohydrates (Rautio et al., 2003). The genus Faecalibacterium includes gram-positive, anaerobic bacteria, which produce butyrate and other short-chain fatty acids through the fermentation of dietary fiber in the host gut (Martín et al., 2017). The Lachnospiraceae (order Clostridiales), degrade plant materials and produce bacteriocins, butyric acid, and lactate (Turnbaugh et al., 2006; Koh et al., 2016). In addition, Lachnospiraceae and Faecalibacterium were associated with improved FCRs and BWG rates in birds (Stanley et al., 2016). Thus, several studies investigating the properties and functions of these bacterial genera have suggested that these microorganisms might be useful as poultry probiotics (Torok et al., 2011; Stanley et al., 2016; Martín et al., 2017).

Similar to the VIRG group, the relative abundances of Lactobacillus in the cecal microbiotas of LPE and HPE groups decreased. These results were consistent with those of Thapa et al. (2012) and Placha et al. (2014), who reported that some 
beneficial commensal bacteria (such as Lactobacillus) were less abundant after PE treatment. In contrast, other studies have shown that PE treatment increased the relative taxon abundance of Lactobacillales (Viveros et al., 2011; Du et al., 2016; Yin et al., 2017; Li et al., 2018). Here, we found that Lactobacillus abundance decreased in the intestine after VIRG treatment. This was consistent with previous studies, which showed that the relative abundance of Lactobacillus decreased in the intestines of chickens after administration of antibiotics (Lan et al., 2005; Guban et al., 2006; Danzeisen et al., 2011; Allen and Stanton, 2014; Mancabelli et al., 2016; Yu et al., 2018). Lactobacillus, which is used as a probiotic, has a beneficial effect on host intestinal health and growth (Huang et al., 2004). However, some Lactobacilli were associated with poor growth performance and FCR (Torok et al., 2011; Stanley et al., 2012). Indeed, some strains of Lactobacillus are sold as weight loss probiotics and others are reported to have the ability to reduce obesity by decreasing appetite and food consumption (Fåk and Bäckhed, 2012). Moreover, De Boever et al. (2000) found that Lactobacillus reuteri decreased lipid absorption and consequently, caused dietary energy losses, possibly by stimulating bacterial bile salt hydrolysis.

The observed shifts in the diversity and composition of the chicken intestinal microbiota may have affected gut microbial metabolism, gut physiological function, host health, and growth. The PICRUSt results showed that, as compared to the CT group, the PE-treated ileal microbiota was enriched in functions associated with cofactors and vitamin metabolism as well as infectious diseases. In addition, PE treatment enriched 15 pathways associated with metabolic function in the cecal microbiota of the LPE group, and 20 pathways associated with metabolic function in the cecal microbiota of the HPE group. These pathways included protein digestion and absorption, amino acid metabolism, lipid biosynthesis proteins, lipopolysaccharide biosynthesis (proteins), citrate cycle (TCA cycle), and lipoic acid metabolism. These results were consistent with those of Li et al. (2018), who reported that essential oils increased protein biosynthesis, as well as lipid and amino acid metabolism, in the colon microbiotas of weaned piglets. Similar alterations in intestinal microbial function may have caused the performance improvements observed in the chickens fed PEs. Several previous studies have also shown that antibiotic treatment affected the predicted functions enriched in the intestinal microbiota (Choi et al., 2017). Danzeisen et al. (2011)

\section{REFERENCES}

Abe, K., Ueki, A., Ohtaki, Y., Kaku, N., Watanabe, K., and Ueki, K. (2012). Anaerocella delicata gen. nov., sp. nov., a strictly anaerobic bacterium in the phylum Bacteroidetes isolated from a methanogenic reactor of cattle farms. J. Gen. Appl. Microbiol. 58, 405-412. doi: 10.2323/jgam.58.405

Allen, H. K., and Stanton, T. B. (2014). Altered egos: antibiotic effects on food animal microbiomes. Annu. Rev. Microbiol. 68, 297-315. doi: 10.1146/annurevmicro-091213-113052

Altschul, S. F., Madden, T. L., Schffer, A. A., Zhang, J., Zhang, Z., Miller, W., et al. (1997). Gapped BLAST and PSI-BLAST: a new generation of protein database search programs. Nucleic Acids Res. 25, 3389-3402. doi: 10.1093/nar/25. 17.3389 demonstrated that Roseburia and Lactobacillus reduced and transport system genes were enriched in the cecal microbiotas of broilers fed VIRG supplements. The current study demonstrated that, similar to VIRG, PEs enhanced broiler growth performance, possibly due to improved intestinal microbial composition and metabolic function.

\section{CONCLUSION}

The results of the present study showed that dietary supplementation with PEs or virginiamycin altered the diversity, composition, and function of the intestinal microbiota. PE treatment enhanced the relative abundances of Firmicutes in duodenal and ileal microbiotas, and the relative abundances of unclassified Rikenellaceae, Faecalibacterium, and Lachnospiraceae in the cecal microbiota. The enhancement of several metabolic pathways in the intestinal microbiota, such as protein digestion/absorption, and amino acid metabolism, might have resulted in the improved chicken growth performance. These results provide a theoretical framework for the use of PEs as an alternative to antibiotics in broiler production.

\section{AUTHOR CONTRIBUTIONS}

$\mathrm{NZ}$ and $\mathrm{BL}$ designed the experiments and wrote the manuscript. JW, KC, and QZ performed the experiments and collected the samples. JW and LY analyzed the data. NZ and BL were responsible for the final content. All of the authors have read and approved the final manuscript.

\section{FUNDING}

This work was supported by the National Natural Science Foundation of China (Project No. 3146068: Beijing, China).

\section{ACKNOWLEDGMENTS}

We are grateful to Prof. Yuan and his co-workers at the Zhuozhou Experiment Station, China Agricultural University for management of the experimental chickens.

Amad, A. A., Männer, K., Wendler, K. R., Neumann, K., and Zentek, J. (2011). Effects of a phytogenic feed additive on growth performance and ileal nutrient digestibility in broiler chickens. Poult. Sci. 90, 2811-2816. doi: 10.3382/ps.201101515

Awaad, M. H. H., Elmenawey, M., and Ahmed, K. A. (2014). Effect of a specific combination of carvacrol, cinnamaldehyde, and Capsicum oleoresin on the growth performance, carcass quality and gut integrity of broiler chickens. Vet. World 7, 284-290. doi: 10.14202/vetworld.2014.284-290

Baser, K. H. C., and Demirci, F. (2007). "Flavours and Fragrances," in Chemistry, Bioprocessing and Sustainability, ed. R. G. Berger (New York, NY: Springer), 43-86.

Bozkurt, M., Küçükyılmaz, K., Çatli, A. U., and Çinar, M. (2009). Effect of dietary mannanoligo -saccharide with or without oregano essential oil and hop-extract 
supplementation on the performance and slaughter characteristics of male broilers. S. Afr. J. Anim. Sci. 39, 223-232.

Brenes, A., and Roura, E. (2010). Essential oils in poultry nutrition: main effects and modes of action. Anim. Feed Sci. Technol. 158, 1-14. doi: 10.1016/j.anifeedsci. 2010.03.007

Burt, S. (2004). Essential oils: their antibacterial properties and potential applications in foods-a review. Int. J. Food Microbiol. 94, 223-253. doi: 10.1016/ j.ijfoodmicro.2004.03.022

Caporaso, J. G., Kuczynski, J., Stombaugh, J., Bittinger, K., Bushman, F. D., Costello, E. K., et al. (2010). QIIME allows analysis of high-throughput community sequencing data. Nat. Methods 7, 335-336.

Chen, H., and Jiang, W. (2014). Application of high-throughput sequencing in understanding human oral microbiome related with health and disease. Front. Microbiol. 5:508. doi: 10.3389/fmicb.2014.00508

Choi, J. H., Lee, K., Kim, D. W., Kil, D. Y., Kim, G. B., and Cha, C. J. (2017). Influence of dietary avilamycin on ileal and cecal microbiota in broiler chickens. Poult. Sci. 97, 970-979. doi: 10.3382/ps/pex360

Ciftici, M., Güler, T., Dalkiliç, B., and Nihat-Ertas, O. (2005). The effect of anise oil (Pimpinella anisum > L.) on broiler performance. Int. J. Poult. Sci. 4, 851-855. doi: 10.3923/ijps.2005.851.855

Cromwell, G. L. (2002). Why and how antibiotics are used in swine production. Anim. Biotechnol. 13, 7-27. doi: 10.1081/abio- 120005767

Danzeisen, J. L., Kim, H. B., Isaacson, R. E., Tu, Z. J., and Johnson, T. J. (2011). Modulations of the chicken cecal microbiome and metagenome in response to anticoccidial and growth promoter treatment. PLoS One 6:e27949. doi: 10.137/ journal.pone.0027949

De Boever, P. D., Wouters, R., Verschaeve, L., Berckmans, P., Schoeters, G., and Verstraete, W. (2000). Protective effect of the bile salt hydrolase-active Lactobacillus reuteri against bile salt cytotoxicity. Appl. Microbiol. Biotechnol. 53, 709-714. doi: 10.1007/s002530000330

DeSantis, T. Z., Hugenholtz, P., Larsen, N., Rojas, M., Brodie, E. L., Keller, K., et al. (2006). Greengenes, a chimera-checked 16S rRNA gene database and workbench compatible with ARB. Appl. Environ. Microbiol. 72, 5069-5072. doi: 10.1128/aem.03006-05

Diarra, M. S., and Malouin, F. (2014). Antibiotics in canadian poultry productions and anticipated alternatives. Front. Microbiol. 5:282. doi: 10.3389/fmicb.2014. 00282

Diaz Carrasco, J. M., Redondo, E. A., Pin Viso, N. D., Redondo, L. M., Farber, M. D., and Fernández Miyakawa, M. E. (2018). Tannins and bacitracin differentially modulate gut microbiota of broiler chickens. Biomed. Res. Int. 2018:11. doi: $10.1155 / 2018 / 1879168$

Diaz Carrasco, J. M., Redondo, L. M., Redondo, E. A., Dominguez, J. E., Chacana, A. P., and Fernandez Miyakawa, M. E. (2016). Use of plant extracts as an effective manner to control clostridium perfringens induced necrotic enteritis in poultry. Biomed. Res. Int. 2016:15.

Dibner, J. J., and Richards, J. D. (2005). Antibiotic growth promoters in agriculture: history and mode of action. Poult. Sci. 84, 634-643. doi: 10.1093/ps/ 84.4.634

Du, E., Wang, W., Gan, L., Li, Z., Guo, S., and Guo, Y. (2016). Effects of thymol and carvacrol supplementation on intestinal integrity and immune responses of broiler chickens challenged with Clostridium perfringens. J. Anim. Sci. Biotechnol. 7, 522-531. doi: 10.1186/s40104-016-0079-7

Edgar, R. C. (2010). Search and clustering orders of magnitude faster than BLAST. Bioinformatics 26, 2460-2461. doi: 10.1093/bioinformatics/btq461

Fåk, F., and Bäckhed, F. (2012). Lactobacillus reuteri prevents diet-induced obesity, but not atherosclerosis, in a strain dependent fashion in Apoe-/- mice. PLoS One 7:e46837. doi: 10.1371/journal.pone.0046837

Gill, S. R., Mihai, P., Deboy, R. T., Eckburg, P. B., Turnbaugh, P. J., and Samuel, B. S. (2006). Metagenomic analysis of the human distal gut microbiome. Science 312, 1355-1359.

Guban, J., Korver, D. R., Allison, G. E., and Tannock, G. W. (2006). Relationship of dietary antimicrobial drug administration with broiler performance, decreased population levels of Lactobacillus salivarius, and reduced bile salt deconjugation in the ileum of broiler chickens. Poult. Sci. 85, 2186-2194. doi: 10.1093/ps/85. 12.2186

Huang, M. K., Choi, Y. J., Houde, R., Lee, J. W., Lee, B., and Zhao, X. (2004). Effects of Lactobacilli and an acidophilic fungus on the production performance and immune responses in broiler chickens. Poult. Sci. 2004, 788-795. doi: $10.1093 / \mathrm{ps} / 83.5 .788$
Jamroz, D., and Kamel, C. (2002). Plant extracts enhance broiler performance. J. Anim. Sci. 80:41.

Jamroz, D., Wiliczkiewicz, A., Wertelecki, T., Orda, J., and Skorupinska, J. (2005). Use of active substances of plant origin in chicken diets based on maize and locally grown cereals. Br. Poult. Sci. 46, 485-493. doi: 10.1080/ 00071660500191056

Jang, I. S., Ko, Y. H., Kang, S. Y., and Lee, C. Y. (2007). Effect of a commercial essential oil on growth performance, digestive enzyme activity and intestinal microflora population in broiler chickens. Anim. Feed Sci. Tech. 134, 304-315. doi: 10.1016/j.anifeedsci.2006.06.009

Kanehisa, M., Goto, S., Sato, Y., Furumichi, M., and Tanabe, M. (2012). KEGG for integration and interpretation of large-scale molecular data sets. Nucleic Acids Res. 40, 109-114. doi: 10.1093/nar/gkr988

Khattak, F., Ronchi, A., Castelli, P., and Sparks, N. (2014). Effects of natural blend of essential oil on growth performance, blood biochemistry, cecal morphology, and carcass quality of broiler chickens. Poult. Sci. 93, 132-137. doi: 10.3382/ps. 2013-03387

Koh, A., De Vadder, F., Kovatcheva-Datchary, P., and Bäckhed, F. (2016). From dietary fiber to host physiology: short-chain fatty acids as key bacterial metabolites. Cell 165, 1332-1345. doi: 10.1016/j.cell.2016.05.041

Lan, Y., Verstegen, M. W. A., Tamminga, S., and Williams, B. A. (2005). The role of the commensal gut microbial community in broiler chickens. World Poult. Sci. J. 61, 95-104. doi: 10.1079/wps200445

Langille, M. G. I., Jesse, Z., Gregory, J., Daniel, M. D., Dan, K., Reyes, J. A., et al. (2013). Predictive functional profiling of microbial communities using 16S rRNA marker gene sequences. Nat. Biotechnol. 31:814. doi: 10.1038/nbt. 2676

Ley, R. E., Knight, R., and Gordon, J. I. (2007). The human microbiome: eliminating the biomedical environmental dichotomy in microbial ecology. Environ. Microbiol. 9, 3-4. doi: 10.1111/j.1462-2920.2006.01222_3.x

Li, Y., Fu, X., Ma, X., Geng, S., Jiang, X., Huang, Q., et al. (2018). Intestinal microbiome-metabolome responses to essential oils in piglets. Front. Microbiol. 9:1988. doi: $10.3389 /$ fmicb. 2018.01988

Louis, P., Hold, G. L., and Flint, H. J. (2014). The gut microbiota, bacterial metabolites and colorectal cancer. Nat. Rev. Microbiol. 12, 661-672. doi: 10. 1038/nrmicro3344

Magoč, T., and Salzberg, S. L. (2011). FLASH: fast length adjustment of short reads to improve genome assemblies. Bioinformatics 27, 2957-2963. doi: 10.1093/ bioinformatics/btr507

Mancabelli, L., Ferrario, C., Milani, C., Mangifesta, M., Turroni, F., Duranti, S., et al. (2016). Insights into the biodiversity of the gut microbiota of broiler chickens. Environ. Microbiol. 18, 884-892. doi: 10.1111/1462-2920. 13363

Martín, R., Miquel, S., Benevides, L., Bridonneau, C., Robert, V., Hudault, S., et al. (2017). Functional characterization of novel Faecalibacterium prausnitzii $>$ strains isolated from healthy volunteers: a step forward in the use of F. prausnitzii > as a next-generation probiotic. Front. Microbiol. 8:1226. doi: 10.3389/fmicb.2017.01226

National Research Council [NRC] (1994). Nutrient Requirements of Poultry, 9th Edn. Washington, D. C: National Academic Press.

Ocak, N., Erener, G., Burak, A. K. F., Sungu, M., Altop, A., and Ozmen, A. (2008). Performance of broilers fed diets supplemented with dry peppermint (Mentha piperita L.) or thyme (Thymus vulgaris L.) leaves as growth promoter source. Czech J Anim Sci. 53, 169-175. doi: 10.17221/373-cjas

Placha, I., Takacova, J., Ryzner, M., Cobanova, K., Laukova, A., Strompfova, V., et al. (2014). Effect of thyme essential oil and selenium on intestine integrity and antioxidant status of broilers. Br. Poult. Sci. 55, 105-114. doi: 10.1080/00071668. 2013.873772

Pourabedin, M., Guan, L., and Zhao, X. (2015). Xylo-oligosaccharides and virginiamycin differentially modulate gut microbial composition in chickens. Microbiome 3, 1-12. doi: 10.1186/s40168-015-0079-4

Ran, C., Hu, J., Liu, W., Liu, Z., He, S., Dan, B. C., et al. (2016). Thymol and carvacrol affect hybrid tilapia through the combination of direct stimulation and an intestinal microbiota-mediated effect: insights from a germ-free Zebrafish model. J. Nutr. 146, 1132-1140. doi: 10.3945/jn.115.22 9377

Rautio, M., Erola, E., Tunkelrott, M. L. V., Molitoris, D., Lawson, P., Collins, M. D. et al. (2003). Reclassification of Bacteroides putredinis $>$ (Weinberg et al., 1937) in a New Genus Alistipes > gen. nov., as Alistipes putredinis > comb. nov., and 
description of Alistipes finegoldii > sp. nov., from human sources. Syst. Appl. Microbiol. 26, 181-188.

Salaheen, S., Kim, S. W., Haley, B. J., Kessel, J. S. V., and Biswas, D. (2017). Alternative growth promoters modulate broiler gut microbiome and enhance body weight gain. Front. Microbiol. 8:2088. doi: 10.3389/fmicb.2017. 02088

Segata, N., Izard, J., Waldron, L., Gevers, D., Miropolsky, L., Garrett, W. S., et al. (2011). Metagenomic biomarker discovery and explanation. Genome Biol. 12:60. doi: 10.1186/gb-2011-12-6-r60

She, Y., Cai, H., and Liu, G. (2018). Effects of antibiotic on microflora in ileum and cecum for broilers by $16 \mathrm{~S}$ rRNA sequence analysis. Anim. Sci. J. 89, 1680-1691. doi: $10.1111 /$ asj.13113

Stanley, D., Denman, S. E., Hughes, R. J., Geier, M. S., Crowley, T. M., Chen, H., et al. (2012). Intestinal microbiota associated with differential feed conversion efficiency in chickens. Appl. Microbiol. Biotechnol. 96, 1361-1369. doi: 10.1007/ s00253-011-3847-5

Stanley, D., Hughes, R. J., Geier, M. S., and Moore, R. J. (2016). Bacteria within the gastrointestinal tract microbiota correlated with improved growth and feed conversion: challenges presented for the identification of performance enhancing probiotic bacteria. Front. Microbiol. 7:187. doi: 10.3389/fmicb.2016. 00187

Stevanović, Z. D., Bošnjak-Neumüller, J., Pajiæ-Lijakoviæ, I., Raj, J., and Vasiljeviæ, M. (2018). Essential oils as feed additives-future perspectives. Molecules 23:1717. doi: 10.3390/molecules23071717

Thapa, D., Losa, R., Zweifel, B., and Wallace, R. J. (2012). Sensitivity of pathogenic and commensal bacteria from the human colon to essential oils. Microbiology 158, 2870-2877. doi: 10.1099/mic.0.061127-0

Torok, V. A., Allison, G. E., Percy, N. J., Ophel-Keller, K., and Hughes, R. J. (2011). Influence of antimicrobial feed additives on broiler commensal posthatch gut microbiota development and performance. Appl. Environ. Microbiol. 77, 3380-3390. doi: 10.1128/AEM.02300-10

Turnbaugh, P. J., Ley, R. E., Mahowald, M. A., Vincent, M., Mardis, E. R., and Gordon, J. I. (2006). An obesity-associated gut microbiome with increased capacity for energy harvest. Nature 444, 1027-1031.

Viveros, A., Chamorro, S., Pizarro, M., Arija, I., Centeno, C., and Brenes, A. (2011). Effects of dietary polyphenol-rich grape products on intestinal microflora and gut morphology in broiler chicks. Poult. Sci. 90, 566-578. doi: 10.3382/ps.201000889

White, J. R., Nagarajan, N., and Pop, M. (2009). Statistical methods for detecting differentially abundant features in clinical metagenomic samples. PLoS Comput. Biol. 5:e1000352. doi: 10.1371/journal.pcbi.1000352
Windisch, W., Schedle, K., Plitzner, C., and Kroismayr, A. (2008). Use of phytogenic products as feed additives for swine and poultry. J. Anim. Sci. 86, 140-148.

Wlodarska, M., Willing, B. P., Bravo, D. M., and Finlay, B. B. (2015). Phytonutrient diet supplementation promotes beneficial Clostridia species and intestinal mucus secretion resulting in protection against enteric infection. Sci. Rep. 5:9253. doi: 10.1038/srep09253

Yin, D., Du, E., Yuan, J., Gao, J., Wang, Y., Aggrey, S. E., et al. (2017). Supplemental thymol and carvacrol increases ileum Lactobacillus population and reduces effect of necrotic enteritis caused by Clostridium perfringes in chickens. Sci. Rep. 7:7334. doi: 10.1038/s41598-017-07420-4

Yu, M., Mu, C., Zhang, C., Yang, Y., Su, Y., and Zhu, W. (2018). Marked response in microbial community and metabolism in the ileum and cecum of suckling piglets after early antibiotics exposure. Front. Microbiol. 9:1166. doi: 10.3389/ fmicb.2018.01166

Zaura, E., Keijser, B. J., Huse, S. M., and Crielaard, W. (2009). Defining the healthy "core microbiome" of oral microbial communities. BMC Microbiol. 9:259. doi: 10.1186/1471-2180-9-259

Zeng, Z., Zhang, S., Wang, H., and Piao, X. (2015). Essential oil and aromatic plants as feed additives in non-ruminant nutrition: a review. J. Anim. Sci. Biotechnol. 6, 7-12. doi: 10.1186/s40104-015-0004-5

Zhang, G. F., Yang, Z. B., Wang, Y., Yang, W. R., Jiang, S. Z., and Gai, G. S. (2009). Effects of ginger root (Zingiber officinale) processed to different particle sizes on growth performance, antioxidant status, and serum metabolites of broiler chickens. Poult. Sci. 88, 2159-2166. doi: 10.3382/ps.2009-00165

Zhao, W., Wang, Y., Liu, S., Huang, J., Zhai, Z., He, C., et al. (2015). The dynamic distribution of porcine microbiota across different ages and gastrointestinal tract segments. PLoS One 10:e0117441. doi: 10.1371/journal.pone.0117441

Conflict of Interest Statement: QZ was employed by Guangdong Ruisheng Technology Co., Ltd.

The remaining authors declare that the research was conducted in the absence of any commercial or financial relationships that could be construed as a potential conflict of interest.

Copyright (c) 2019 Zhu, Wang, Yu, Zhang, Chen and Liu. This is an open-access article distributed under the terms of the Creative Commons Attribution License (CC BY). The use, distribution or reproduction in other forums is permitted, provided the original author(s) and the copyright owner(s) are credited and that the original publication in this journal is cited, in accordance with accepted academic practice. No use, distribution or reproduction is permitted which does not comply with these terms. 